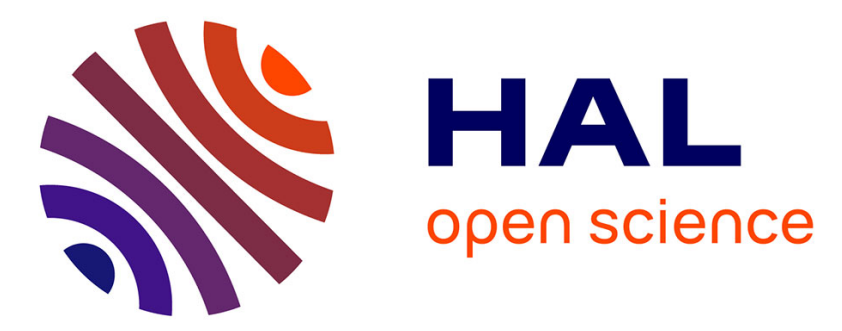

\title{
Chronique: Dons à la photothèque de l'Ecole - Le bois se porte bien - Assemblée générale de la commission internationale de la lutte biologique à Tunis - Les cahiers du centre technique du bois
}

- [.]revue Forestière Française, Rédaction

\section{- To cite this version:}

- [.]revue Forestière Française, Rédaction. Chronique: Dons à la photothèque de l'Ecole - Le bois se porte bien - Assemblée générale de la commission internationale de la lutte biologique à Tunis - Les cahiers du centre technique du bois. 1962, pp.433. 10.4267/2042/24467 . hal-03535230

\author{
HAL Id: hal-03535230 \\ https://hal.science/hal-03535230
}

Submitted on 19 Jan 2022

HAL is a multi-disciplinary open access archive for the deposit and dissemination of scientific research documents, whether they are published or not. The documents may come from teaching and research institutions in France or abroad, or from public or private research centers.
L'archive ouverte pluridisciplinaire HAL, est destinée au dépôt et à la diffusion de documents scientifiques de niveau recherche, publiés ou non, émanant des établissements d'enseignement et de recherche français ou étrangers, des laboratoires publics ou privés. 


\section{CHRONIQUE}

\section{Le bois se porte bien}

La construction dans la plupart des cités modernes d'innombrables immeubles à charpente métallique, à huisserie métallique, à parquets de béton couverts de revêtement plastique, la publicite faite à tel ou tei grand transatlantique pour vanter l'absence totale de produits ligneux à son bord, la vogue passagère des traverses de chemin de fer en métal ou en béton, celle beaucoup plus durable du soutènement métallique dans la plupart des mines ou des poteaux en ciment armé ou en acier dans le transport et la distribution de l'énergie électrique amènent souvent à penser que le bois est un matériau périmé dont les besoins vont en déclinant d'une façon irrémédiable et dont, conclusion logique d'un jugement hâtif, il n'y a pas lieu pour les pouvoirs publics de favoriser la production.

Or, bien loin d'avoìr à craindre une surproduction de bois, il est à peu près certain que le monde s'achemine vers une pénurie de ce matériau, d'autant plus grave que la production ligneuse est une production à longue échéance, et que la satisfaction des besoins de l'an 2000 dépend d'une façon inéluctable de décisions à prendre et d'investissements à réaliser dès maintenant.

Les statistiques de la F.A.O. pour l'année 1960 sont à cet égard très convaincantes.

La valeur de la production ligneuse mondiale en 1960 a été estimée à 178 milliards de nouveaux francs, en augmentation de 18 milliards par rapport à l'année précédente.

En volume, l'évolution de la production forestière a été la suivante pour les diverses catégories de produits (chiffres donnés en milliers $\mathrm{de}^{3}$ ):

\begin{tabular}{|c|c|c|}
\hline & 1959 & 1960 \\
\hline Bois d'œuvre résineux. & $\overline{468715}$ & $4 \overline{2} 155$ \\
\hline feuillus .. & 143255 & 149455 \\
\hline $\begin{array}{r}\text { Bois d'industrie résineux } \ldots \ldots \ldots \ldots \\
\text { fenillus } \ldots \ldots \ldots \ldots . .\end{array}$ & $\begin{array}{r}270195 \\
79410\end{array}$ & $\begin{array}{r}272155 \\
85215\end{array}$ \\
\hline $\begin{array}{r}\text { Bois de chauffage résineux } \\
\qquad \text { feuillus } \ldots \ldots \ldots . . .\end{array}$ & $\begin{array}{l}695460 \\
148140\end{array}$ & $\begin{array}{l}672965 \\
135505\end{array}$ \\
\hline
\end{tabular}

Le bois de chauffage mis à part, l'augmentation de la production est absolument générale; elle apparaitrait encore plus importante pour le bois d'industrie, si l'essor remarquable du bois de raperie n'était en partie compensé par un certain déclin des bois de mine et des poteaux de ligne.

La production de sciages qui, dans l'esprit de beaucoup, serait en train de péricliter au bénéfice de nouveaux modes d'utilisation du bois, s'est élevée au chiffre jamais atteint de 330 millions de $\mathrm{m}^{3}$ (soit 7 millions de plus qu'en 1959) se répartissant en 263 millions de $\mathrm{m}^{3}$ de résineux et 67 millions de $\mathrm{m}^{3}$ de feuillus. 
Tous les principaux producteurs de bois résinet $\bar{x}$ ont notablement accru leurs exportations par rapport à l'année précédente: le Canada de $970000 \mathrm{~m}^{3}$, la Finlande de $860000 \mathrm{~m}^{3}$, la Suède de $344000 \mathrm{~m}^{3}$, la France de $334000 \mathrm{~m}^{3}$, la Roumanie enfin de $231000 \mathrm{~m}^{3}$.

L'Union Soviétique représente de plus en plus le principal fournisseur de bois résineux; sa production s'est élevée en 1960 à 91 millions de $\mathrm{m}^{3}$ représentant $34 \%$ de la production mondiale, en augmentation sur 1959 de 1710000 $\mathrm{m}^{3}$.

En Amérique du Nord au contraire, la production de sciage résineux a diminué de $1855000 \mathrm{~m}^{3}$; les Etats-Unis, deuxième producteur mondial, bien qu'ayant réduit leurs importations de $343000 \mathrm{~m}^{3}$, continuent à tenir la première place en tant qu'acheteur de produits ligneux avec une importation totale de $9308000 \mathrm{~m}^{3}$.

De 1959 à 1960, la part des pays européens dans le commerce international des bois résineux est passée de 47 à $48 \%$ à l'exportation et de 43 à $62 \%$ à l'importation.

Le Royaume-Uni demeure le deuxième importateur mondial de sciages résineux, ayant acheté à l'étranger en $19608550000 \mathrm{~m}^{3}$, en augmentation de $24 \%$ sur l'année précédente.

En ce qui concerne les bois durs, les pays européens demeurent les principaux exportateurs avec $37 \%$ des exportations mondiales, en augmentation de $410000 \mathrm{~m}^{3}$ par rapport à 1959 .

Les principaux exportateurs européens ont été dans l'ordre la Roumanie avec $494000 \mathrm{~m}^{3}$, la France avec $379000 \mathrm{~m}^{3}$ (en augmentation de $39 \%$ sur l'année précédente) et la Yougoslavie avec $378000 \mathrm{~m}^{3}$.

Les pays asiatiques ont également augmenté leur production de bois dur de $255000 \mathrm{~m}^{3}$ par rapport à 1959; ces pays livrent $32 \%$ du total des exportations mondiales, et la part prise sur le marché par certains d'entre eux est en large progrès: les exportations malaises ont fait un véritable bond avec $412000 \mathrm{~m}^{3}$ en 1960 , en augmentation de $55 \%$ par rapport à l'année précédente, la Malaisie devenant ainsi le second exportateur mondial de bois dur; la Thailande a accru également ses exportations de $55 \%$ et le petit état de Sarawak en Indonésie de $35 \%$.

L'Union Soviétique qui est surtout connue en tant que fournisseur de bois résineux est également un important producteur de sciages feuillus; sa production est estimée à 16 millions de $\mathrm{m}^{3}$, soit $24 \%$ du total mondial.

Quant aux Etats-Unis, ils ont produit en 196014,8 millions de $\mathrm{m}^{3}$ (23\% de la production mondiale), en augmentation de $13 \%$ par rapport à 1959.

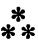

Les statistiques de la F.A.O. font ensuite le point de la production des produits ligneux qui paraissent bénéficier actuellement de la plus grande expansion: contreplaqués, panneaux de fibres et panneaux de particules.

La production mondiale de contreplaqué s'est élevée, en 1960, à 15,4 millions de $\mathrm{m}^{3}$, en augmentation de $3,5 \%$ sur l'année précédente. Les principaux producteurs ont été les Etats-Unis, avec 7,9 millions de $\mathrm{m}^{3}$, le Japon avec 1,29 million de $\mathrm{m}^{3}$, l'Union Soviétique avec 1,35 million de $\mathrm{m}^{3}$ et le Canada de 0,97 million de $\mathrm{m}^{3}$. En Europe, le principal producteur était, en 1960, l'Allemagne Fédérale $\left(0,66\right.$ million de $\left.\mathrm{m}^{3}\right)$. La France, bien que favorisée au point de vue matière première grâce à un approvisionnement aisé en okoumé en provenance des territoires africains de la Communauté et en 

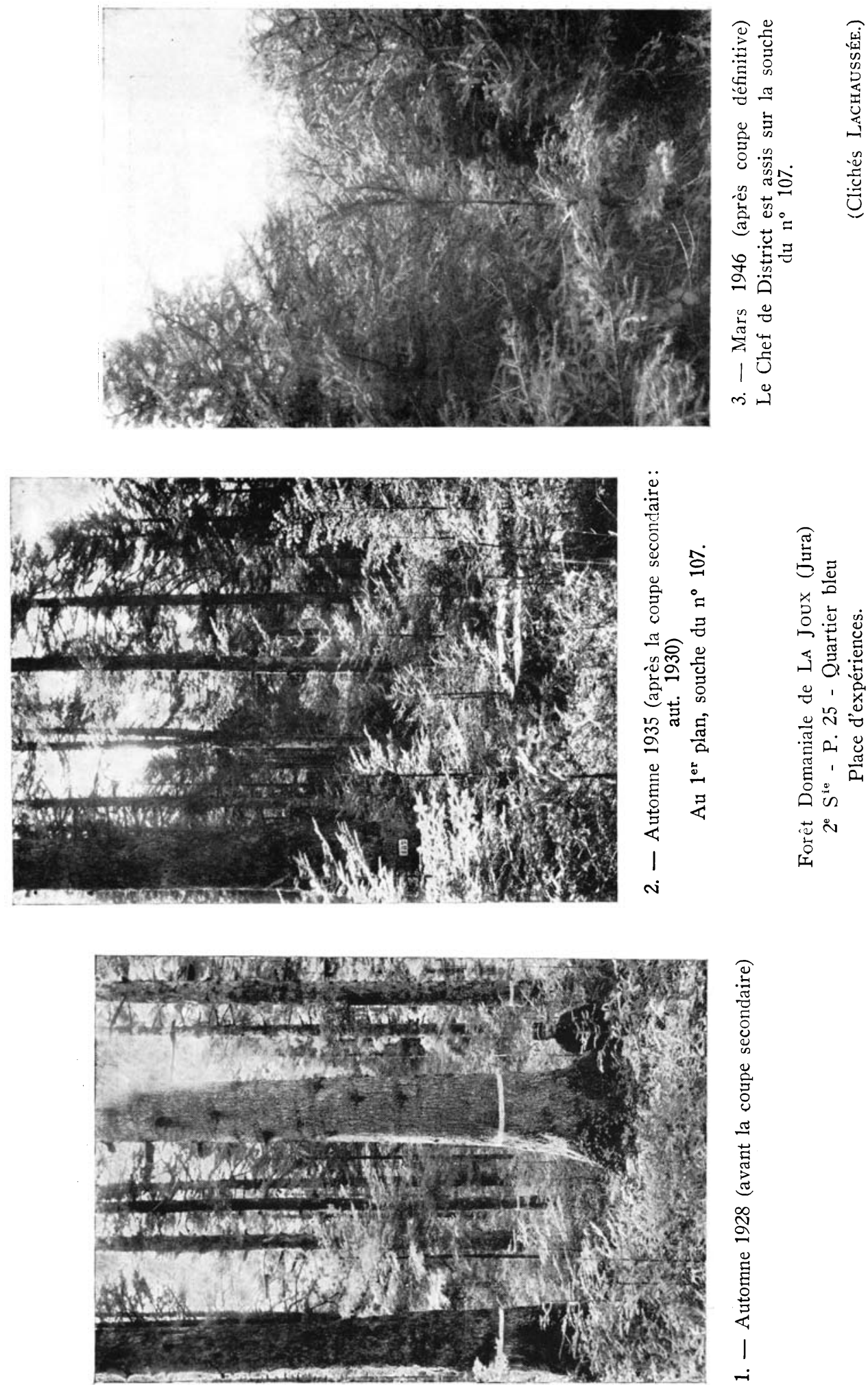

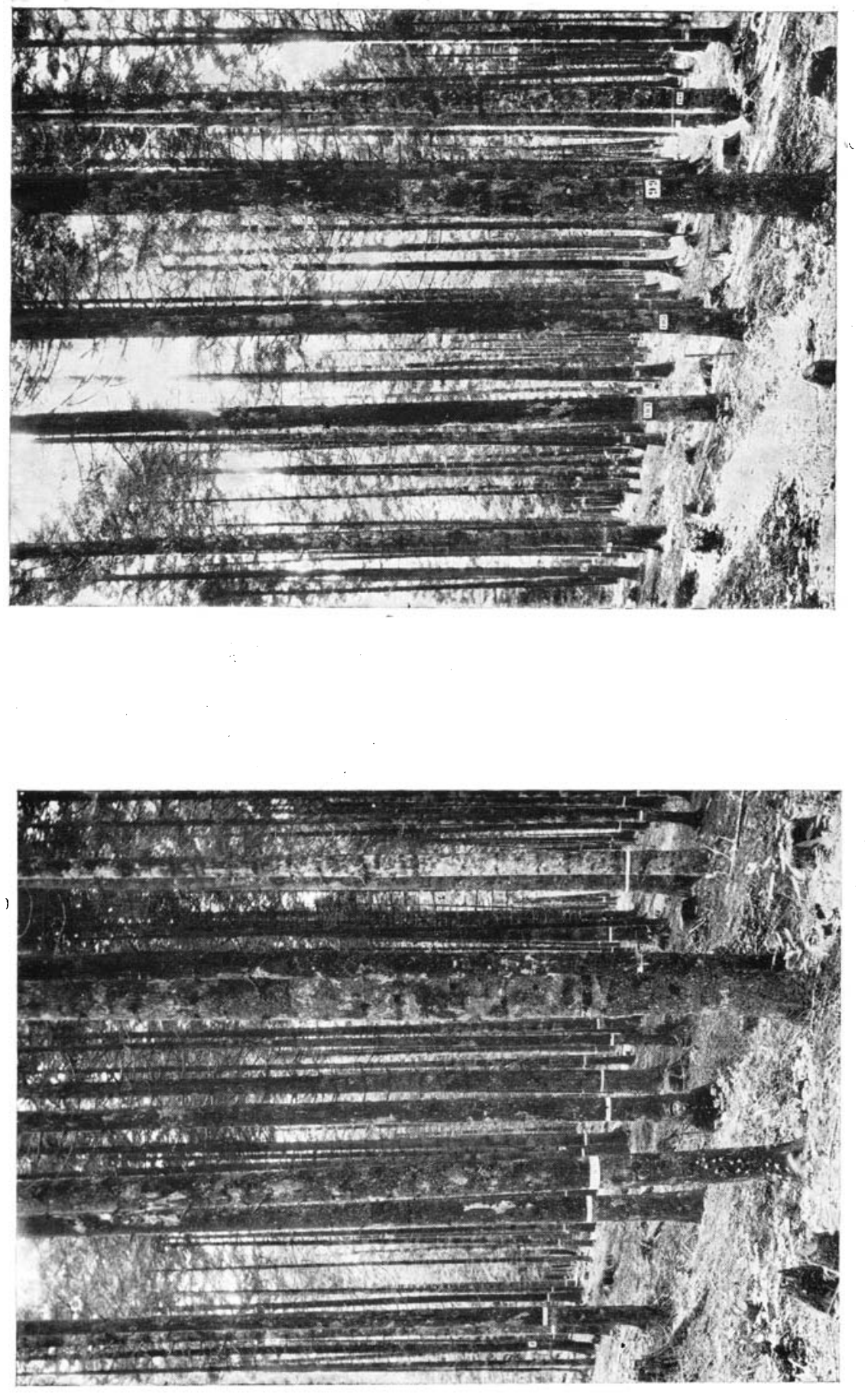

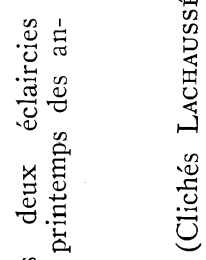

总弟

눙

เก

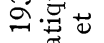

봅요

욤욤

।

ن

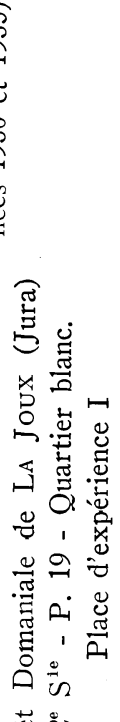

苛

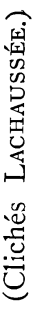

.

ษ

范

芯

్ㅣㅁ

岂

冚

I 
hêtre métropolitain, ne produit que 0,4 million de $\mathrm{m}^{3}$ de bois contreplaqué: mais il s'agit d'une production en expansion constante et dont une part importante $(18 \%)$ est vendue à l'étranger. Le Royaume-Uni demeure le principal acheteur de contreplaqué,. ses importations ayant porté en 1960 sur $640000 \mathrm{~m}^{3}$, en augmentation de $30 \%$ par rapport à l'année précédente.

La production mondiale de panneaux de fibres a été en 1960 de 4,26 millions de tonnes, en augmentation de $4,8 \%$, la production française étant elle-même passée de 112000 à 130000 tonnes, soit une augmentation de $20 \%$.

L'expansion est encore plus importante sur les panneaux de particules dont la production mondiale a augmenté de 1100000 tonnes en 1959 à 1463000 en 1960 (soit $33 \%$ de plus), la production française jouissant quant à elle d'une expansion supérieure à la moyenne mondiale, puisqu'elle est passée de 102000 tonnes en 1959 à 138000 tonnes en 1960, soit plus de $35 \%$ d'augmentation.

A moins d'un retournement bien improbable de la conjoncture, de telles statistiques montrent, malgré l'aridité des chiffres, que de sérieux problèmes d'approvisionnement risquent de se poser, non seulement, on le sait déjà, pour l'industrie papetière, mais également pour la plupart des autres industries utilisant le bois.

H. $\mathbf{P}$.

\section{Dons à la Photothèque de l'Ecole}

Le 23 février, M. l'Ingénieur Général LAchaussée a fait don à la Photothèque de l'Ecole d'une collection de 337 clichés pris au cours de sa carrière dans le Jura et le Massif Central. Sa présentation précise et méthodique en rend l'examen particulièrement aisé. Elle comprend:

- un catalogue, dans l'ordre des forêts ou des sujets;

- les plaques négatives rangées en boîtes;

- des albums où sont présentés les tirages ou leurs agrandissements avec les légendes correspondantes.

On y trouve notamment:

- une série de photographies prises dans les places d'expériences de la forêt domaniale de la Joux, de 1928 à 1946;

- une série de photographies prises dans l'arboretum de la Joux, de 1924 à 1948.

C'est ainsi que quelques photographies ont pu être prises au même emplacement et avec le même appareil à des intervalles de 5,10 ou 20 ans.

La Photothèque de l'Ecole s'enrichit ainsi d'un apport précieux et possède désormais une documentation photographique de premier ordre sur le Jura.

Que M. l'Ingénieur Général LachausséE veuille bien trouver ici l'expression des plus vifs remerciements de la Direction et du corps enseignant de l'Ecole, et plus spécialement du service de Documentation.

Et puisse son exemple être suivi! Comme la Bibliothèque de 1'Ecole, dont la richesse dépend en grande partie des dons et legs qu'elle a reçus, la constitution d'une Photothèque est une œuvre collective.

Que ceux qui ont eu l'occasion de réunir, au cours de leur carrière, une collection de clichés - qu'ils soient noirs ou en couleur - pensent à en faire don à la Photothèque de l'Ecole où ils seront méthodiquement classés et répertoriés sur cartes perforées (par sujet, par essence et par lieu géographique). Ils contribueront ainsi très efficacement à enrichir un instrument de travail unique en France, mis à la disposition des jeunes générations de forestiers.

M. MotTe. 


\section{Assemblée générale de la Commission Internationale de Lutte Biologique à Tunis}

L'Assemblée Générale triennale de la C.I.L.B. a eu lieu à Tunis du 25 mars au 6 avril 1962.

On sait que cette Commission a pour but de promouvoir, coordonner et intensifier, pour un meilleur rendement, les recherches et les applications de la lutte biologique contre les insectes et les plantes nuisibles à l'Agriculture des pays ayant des adhérents.

Pour y parvenir, une dizaine de groupes de travail comprenant les chercheurs de divers pays et de divers organismes de recherche ont été créés parmi lesquels nous citerons ceux de la « mouche de l'olive », de la « cochenille des agrumes 》, de la « mouche de la datte 》, et surtout ceux des 《défoliateurs forestiers 》 et 《des insectes du peuplier 》.

Quatorze nations membres étaient représentées à l'Assemblée ; dans chacune un ou plusieurs organismes gouvernementaux cotisent à la Commission. C'est ainsi que pour la France il y a le ministère de l'Agriculture (Protection des Végétaux), l'Institut National de la Recherche Agronomique, la Station de Recherches Forestières et le Service de Défense des Végétaux d'Algérie. La délégation française était composée de sept membres parmi lesquels M. Trouvelot représentait la Recherche Agronomique, M. Maury, Ingénieur en Chef des Eaux et Forêts, la Recherche Forestière, M. Bouyx, la Protection des Végétaux, M: Weill, le ministère de l'Agriculture.

Présidée par M. le Professeur Balachowsky de l'Institut Pasteur et avec comme Secrétaire Général M. Grison, Chef du Laboratoire de Biocoenotique et de Lutte Biologique de l'Institut National de la Recherche Agronomique, l'Assemblée a pris diverses résolutions contenant des recommandations aux Etats membres. Elle a procédé à divers aménagements intérieurs à son organisation et elle a renouvelé son bureau en réélisant le Président et le Secrétaire Général ainsi que d'autres membres, M. le Docteur Pavan (Italie) et M. Romanyk (Espagne) représentant les forestiers au bureau exécutif. M. Knani, Ministre de l'Agriculture de la.République Tunisienne, a ouvèrt les réunions par un exposé technique montrant une rare compétence et une égale compréhension du sens et des méthodes de la Lutte Biologique.

Après la réunion, une visite complète dı pays a amené les membres depuis les orangeraies du Nord (Hammamet) en passant par les oliveraies du Sud (Sfax) jusqu'aux oasis de l'extrême Sud (Gabès, Zarzis, Djerba) et enfin une excursion, réservée plus spécialement aux forestiers, les a amenés en Khroumirie (Le Bélif, Tabarka, Aïn Draham, Fernana) visiter les suberaies attaquées par Lymantria dispar ainsi que les pins maritimes victimes de la Processionnaire.

Parallèlement à ces visites, les représentants du Service forestier local et les membres de la Station de Recherches Forestières tunisienne ont permis aux forestiers d'avoir un panorama plus large de la foresterie tunisienne: les réalisations en matière de dunes, de reboisement, de DRS ont été présentées aux participants. Nous n'oublierons pas. quant à nous. une remarquable visite du Cap Bon ainsi que celle des peuplements naturels surprenants de chênes Kermès de la région de Tabarka qui atteignent dix mètres de haut, où nous avons été conduits par M. Schoenenderger de la Station de Recherches Forestières. L'équipement de celle-ci ne le cède en rien et dépasse souvent les organismes semblables en France.

Accueillis avec empressement par d'anciens élèves tunisiens de l'Ecole forestière, ces tournées ont permis de constater que la camaraderie traditionnelle des forestiers maintient toujours étroits, en dépit de bien des vicissitudes, les liens créés par une même formation et un même amour du métier. 
Nos remerciements vont plus particulièrement à M. HAmzA (131 ${ }^{\circ}$ promotion - Nancy), Ingénieur Principal des Eaux et Forêts à Béja, aux Ingénieurs des Travaux Ben Salah (68 promotion - Les Barres) à Tabarka, et Ben Djenana (68 promotion - Les Barres) à Ain Draham, dont nous n'oublierons pas la réception.

Nous ne pouvons achever ce compte rendu sans signaler notre admiration pour le dynamisme dont fait preuve partout le jeune service forestier tunisien et pour l'organisation de diverses manifestations de l'Assemblée due à M: le - Professeur Jamoussi de l'Ecole súpérieure d'Agriculture de Tunis.

R. MaUry.

\title{
Les cahiers du Centre Technique du Bois
}

\author{
10 , avenue de Saint-Mandé - Paris $\left(12^{\mathrm{e}}\right)$
}

Le Centre Technique du Bois vient de publier son cahier $n^{0} 50$ : «Tableaux d'utilisation en flexion des Bois de construction de section rectangulaire (Bois. résineux).

Les bois sont très fréquemment employés, dans la construction, sous forme de sciages rectangulaires. Ces bois sont presque toujours soumis à des efforts de flexion et la tradition conduit, pour des raisons de 'sécurité, à utiliser des sections généralement surabondantes. 'Mais, dans les conditions actuelles, il est nécessaire de rechercher des économies, et il faut éviter, d'autre part, d'adopter des sections trop faibles dont l'emploi peut entraîner des déformations exagérées.

M. F.-X. Brochard, Ingénieur des Travaux Publics, Ingénieur-Conseil au C.T.B., a donc mis au point avec le concours de M.-J. LANEN, des tableaux d'emploi en flexion des principales sections de bois résineux.

Dans un premier chapitre, les notions élémentaires de résistance des matériaux ont été résumées. Un deuxième chapitre contient les principales formules pratiques de la résistance des matériaux en flexion simple, présentées sous une forme nouvelle et adaptées au matériau «bois 》. Ces formules sont complétées par des exemples. Enfin, le troisième chapitre, comporte 28 tableaux d'utilisation des bois rectangulaires, pour différents eas de charges et différents types de sollicitations.

Il est hors de doute que ce cahier rendra les plus grands services à tous les architectes, ingénieurs, entrepreneurs et charpentiers, qui utilisent le bois et qu'il leur permettra de l'employer dans des conditions rationnelles. 